\title{
Variable cerebral responses to equally distinct deviance in four auditory dimensions: A mismatch negativity study
}

\author{
LEON Y. DEOUELL ${ }^{a}$ AND SHLOMO BENTIN ${ }^{\mathrm{a}, \mathrm{b}}$
}

${ }^{a}$ Department of Psychology, Hebrew University of Jerusalem, Israel

${ }^{\mathrm{b}}$ Center for Neural Computation, Hebrew University of Jerusalem, Israel

\begin{abstract}
In this study we compared the amplitude, latency, and spatial distribution of the mismatch negativity (MMN), elicited by tones deviating in either frequency, intensity, stimulus-onset asynchrony (SOA) or location, and assessed inter- and intra-subject variability. Cross-dimensional comparisons were facilitated by adjusting the magnitude of deviance individually, so that the detection rates were similar, both across dimensions (within subject) and across subjects (within dimension). Despite similar detection rates, the MMN elicited by frequency deviance was larger, and the MMN elicited by SOA deviance was earlier than the other types of MMN. The reliability of frequency-related MMN was better than that of the other types of MMN. The results highlight the problems of comparing MMN across dimensions, especially in clinical populations.
\end{abstract}

Descriptors: Auditory discrimination, Event-related potentials, Mismatch negativity, Auditory attention, Clinical application

Mismatch negativity (MMN) is an event-related potential (ERP) component elicited by a "deviant" stimulus presented infrequently in a train of repeated "standard" stimuli. Deviance may occur in different dimensions. For example, the deviant stimulus may differ from the standard in frequency (e.g., Sams, Paavilainen, Alho, \& Näätänen, 1985), intensity (e.g., Näätänen, Paavilainen, Alho, Reinikainen, \& Sams, 1987), inter-stimulus interval (e.g., Näätänen, Jiang, Lavikainen, Reinikainen, \& Paavilainen, 1993), duration (e.g., Paavilainen, Alho, Reinikainen, Sams, \& Näätänen, 1991), spatial location (e.g., Paavilainen, Karlsson, Reinikainen, \& Näätänen, 1989), phonetic value (e.g., Aaltonen, Niemi, Nyrke, \& Tuhkanen, 1987), or more complex temporal structure (e.g., Schröger, Tervaniemi, \& Näätänen, 1995). Recent studies demonstrated indeed that the MMN elicited by deviance in different dimensions may rely not only on the unified representation of the stimulus but also on the representation of distinct auditory features embedded in the echoic trace (Deacon, Nousak, Pilotti, Ritter, \& Yang, 1998; Giard et al., 1995; Schröger, 1995; for a review see Ritter, Deacon, Gomes, Javitt, \& Vaughan, 1995).

An important characteristic of the MMN paradigm is that the attention of the subject does not have to be focused on the stimuli. Therefore, the MMN is considered to be associated with a pre-

This study has been supported by a grant from the Israel Science Foundation, founded by the Israel Academy of Sciences and Humanities, and by a European Union grant BMH4-CT96-0819 to COBRAIN project.

We thank Prof. Walter Ritter and Prof. Erich Schröger, for helpful comments on a previous version of this manuscript, and Ms. Naomi Wexler for skillful assistance.

Address reprint requests to: Dr. Leon Deouell, Department of Psychology, Hebrew University, Jerusalem, 91905, Israel. E-mail: msleon@ mscc. huji.ac.il. attentive detection of a mismatch between an incoming stimulus and the echoic memory trace formed by the standard stimulus (Näätänen, 1990, 1992). Because the detection of the difference between the standard and the deviant depends on the ability of the auditory system to decipher (and encode) the relevant specific dimension, the MMN may be used not only as a tool for studying echoic memory (cf., Ritter et al., 1995), but also as a physiological sign for the encoding of specific stimulus attributes. Thus, differences in the amplitude, latency, and spatial distribution of the MMN elicited by manipulating different dimensions may be used to compare the integrity of the auditory system involved in processing those dimensions. Such comparisons may provide insights into various normal conditions (e.g., with varied levels of attention involved, in combination with stimulation in another modality) and pathological conditions such as developmental disabilities, stroke, and degenerative diseases (cf. Aaltonen et al., 1987; Korpilahti \& Lang, 1994; Kraus \& McGee, 1994). The use of MMN (rather than performance measures) is particularly advantageous for testing individuals who cannot provide performance data, such as patients with unilateral neglect or patients in coma (Kane, Curry, Buttler, \& Cummins, 1993). Moreover, using MMN may help disentangle pure auditory from higher-level mechanisms. A prerequisite of this approach, however, is to establish the normal pattern of the MMN and its variability across dimensions of deviance. Accordingly, the purpose of the present study was not to discern differences in processing efficiency across dimensions but rather, keeping perceptual efficiency and experimental conditions constant, to examine the dimension-related MMN characteristics.

Reliable use of MMN for comparing the distinct processing of individual stimulus features is hindered by two major impediments. The first is the high sensitivity of the MMN to experimental design. Factors such as interstimulus interval (ISI), intensity of the 
stimuli, characteristics of the standard, and probability of the deviance may affect the observed amplitude and latency of the MMN significantly (see Näätänen, 1992, pp. 148-160 for a review). Consequently, in the absence of a standardized design, comparisons between experiments are risky even when the same dimension of deviance is explored. This problem is even more conspicuous when MMNs elicited by deviance in different dimensions are compared, because it is possible that each of the above factors interacts differently with different dimensions of deviance. The few studies that have attempted to examine these interactions have revealed their complexity. For example, it has been shown that whereas the MMN elicited by deviance in frequency is independent of the intensity, the ISI, or of the duration of tones, the MMN elicited by deviation in intensity is directly and linearly correlated with the absolute tone intensity, the duration of the tones, and the ISI between the tones (Böttcher-Gandor \& Ullsperger, 1992; Paavilainen, Jiang, Lavikainen, \& Näätänen, 1993; Schröger, 1994, 1996). The interaction between other experimental factors and different dimensions of deviance is as yet unknown. In the absence of such knowledge, the effect of manipulating the dimension of deviance may be easily confounded with differences induced by its possible interaction with local experimental factors. This complexity is augmented by the considerable intrasubject test-retest variability of MMN, which may limit the interpretations of within-subject differences among blocks in the same experiment (Escera \& Grau, 1996; Lang et al., 1995; Pekkonen, Rinne, \& Näätänen, 1995). One way to minimize this irrelevant variance is to compare the MMN elicited by deviants in different auditory dimensions within subject while the different deviants are intermixed within block.

The second impediment to comparing the MMN associated with various stimulus dimensions is that the subjects' psychophysical discrimination level may differ across dimensions. Moreover, the pattern of these differences is not necessarily the same across subjects. A specific change in frequency, for example, may be easily detected by one person and hardly noticeable for another, whereas the opposite may be true for shifts in spatial location of the sound source. This problem is troublesome because of the correlation between individual discrimination capacity and MMN (Kraus et al., 1993; Lang et al., 1990; Näätänen, Schröger, Tervaniemi, Karakas, \& Paavilainen, 1993; Sams et al., 1985), as well as the correlation between the amplitude and latency of the MMN and the magnitude of difference between standard and deviant stimuli (Näätänen, Paavilainen, Alho, Reinikainen, \& Sams, 1989; Paavilainen et al., 1989; Tiitinen, May, Reinikainen, \& Näätänen, 1994). Therefore, a reliable comparison of MMN across dimensions must be based on prior adjustment of the magnitude of deviance in each dimension to the individual subject's psychophysical performance. This way, emerging differences across dimensions could be ascribed to characteristics related to processing of a specific dimension, rather than to individual differences in the ability to detect changes in specific dimensions. Whereas electrophysiological data were previously reported along with behavioral discrimination data, this has been done only at the group level (e.g., Sams et al., 1985; Schröger, 1995, Schröger \& Wolff, 1997). Moreover, these studies confirmed that subjects vary widely in their ability to detect the deviant stimuli. To our knowledge, adaptation of the amount of deviance in different dimensions according to each subject's subjective discrimination threshold has not yet been reported.

In the present study, we compared the MMN elicited by deviance in different dimensions of tones while addressing some of the above problems. Recently, Deacon et al. (1998) reported that when deviants along three different dimensions were intermixed in one block, the MMN elicited by each type of deviant seemed to be independent of the existence of other deviants in the block. On the basis of this finding, we compared the MMN elicited by deviance in frequency, intensity, stimulus-onset asynchrony (SOA), or spatial location, intermixed in the same blocks. We took special care to match the magnitude of the difference between standard and deviant stimuli across dimensions, by adjusting the magnitude of each deviance to the individual discrimination threshold of the subject in the respective dimension. Furthermore, because the auditory deficits of some patients may be asymmetrically lateralized, the effect of different dimensions of deviance was explored by comparing the MMN elicited by deviants presented to the right or the left side of the subject. Finally, because keeping the subjective deviance constant led to variability in the objective deviance across subjects, we were able to correlate the amplitude and the latency of each MMN to the objective physical difference between standards and deviants. If significant correlations were found despite the subjectively equated deviance, a role for the objective magnitude of deviance in modulating the MMN could not be discarded. In addition, we calculated the correlation between the MMN obtained in response to deviance in different dimensions. This correlation may be important in clinical practice in which attempts are made to find differential deficits in processing different dimensions within single patients. Given the high variability of the MMN between subjects, a significant within-subjects correlation between dimensions would entail that a subject presenting with a "good" MMN in one dimension should be expected to present with a "good" MMN in other dimensions.

\section{Methods and Materials}

\section{Subjects}

Eight undergraduate students (four female and four male) consented to participate in the study following detailed description of the procedure and general aims. They were paid for participation. All subjects were right-handed and had no known hearing loss.

\section{Stimuli}

Standard stimuli

The standard stimuli were $932 \mathrm{~Hz}, 70 \mathrm{~dB}$ (SPL), $100 \mathrm{~ms}$ long (10 $\mathrm{ms}$ rise and fall time) pure tones. The stimuli were presented from a loudspeaker placed $60^{\circ}$ to the right or to the left of the subjects' midsagittal plane. The SOA between standards was $500 \mathrm{~ms}$.

\section{Deviant stimuli}

There were four types of deviant tones in each block. The deviant tones differed from the standard tones in that they either had a lower frequency, or a softer intensity, or their source had a more medial location or the SOA between them and the preceding standard tones was shorter than the SOA between two standard tones. The magnitude of deviation in each of these four features was determined for each subject individually using the following procedure:

Stage 1. A staircase method was used to determine the minimal magnitude of difference between the standard and deviant that was clearly noticeable. For frequency, intensity, and source-location, the subject was presented with consecutive pairs of stimuli, the first of which was always the standard tone, as characterized above. The subject had to decide whether the second stimulus was iden- 
tical to or different from the standard in a predesignated dimension. If a difference was not detected, it was increased in the following trial, by changing the value of the second tone by one predetermined step in the tested dimension. When the difference was detected, it was decreased in the same manner. For determining the SOA deviance level, the same procedure was used, except that the subject was presented with five stimuli (i.e., four ISIs) instead of two, and had to say whether the fourth interval was the same as those intervals preceding it. The steps were $2 \mathrm{~Hz}$ for frequency, $1 \mathrm{~dB}$ for intensity, $10 \mathrm{~ms}$ for $\mathrm{SOA}$, and $15^{\circ}$ for location. The procedure was continued until the staircase undulated repeatedly within a certain range. This range was determined twice for each subject and each dimension, once starting from no difference and once starting a from a large difference. For each dimension, the magnitude of deviance to be used while recording the ERPs was selected from the higher limit of the determined range, that is, a deviance that was consistently detected. The selected value was tested in the second stage.

Stage 2. Following the staircase stage, the detection accuracy of the selected difference magnitude was tested. For each dimension, the subject was presented with 100 groups of four consecutive tones at $500 \mathrm{~ms}$ SOA. Half of these quartets consisted of four identical standard tones and half consisted of three standard tones followed by a deviant tone differing from the standard in one dimension. The magnitude of the deviance was that selected in the first stage. The subject's task was to press one button if the four tones were identical and another if the last tone differed from the first three. If the hit rate for detecting the deviant tones was lower than $95 \%$ or the false alarm rate higher than $10 \%$ (or $d^{\prime}$ over 2.9 ), stage 2 was repeated using a larger difference.

Stage 3. At this stage, deviant tones along all four dimensions were presented intermixed with the standards in one block, at 500 ms SOA (except for SOA-deviants). Twenty-five deviants of each type were randomly interspersed among 400 standard stimuli and the subjects were instructed to press a button whenever a deviant occurred, regardless of deviance type. Once more, adjustments were made if a deviance was detected less than $92 \%$ of the time (2 misses out of 25 trials) or false alarms were more than $10 \%$. In fact, false alarms were rare at this stage.

To conclude, the magnitude of deviance selected for each deviance type was the magnitude at which discrimination performance showed a $d^{\prime}$ of over 2.9 in stage 2, (where $d^{\prime}$ could be accurately computed) and a hit rate of over $92 \%$ in stage 3 . The resulting magnitudes of deviance in each dimension for each subject are presented in Table $1 .^{1}$

\section{Procedure}

Following a preliminary session in which the deviance magnitudes were determined, the electroencephalogram (EEG) was recorded in two sessions, each on a separate day. The entire set was never spread over a period of more than 1 month. Four subjects were tested with stimuli presented on their left side in the first session

${ }^{1}$ The correlation between the individual deviance in the SOA and intensity dimensions was statistically significant (Pearson's $r=.75, p=$ $.032)$, as was the correlation between SOA and frequency $(r=.83, p=$ $.011)$. Although the same trend was found for the correlation between pitch and intensity dimensions, it did not reach statistical significance $(r=.558$, $p=.151$ ). As there was practically no variability in the location dimension, there was no point in testing the correlation with this variable.
Table 1. Individual Magnitudes of Deviance for Pitch, Intensity, SOA, and Location ${ }^{a}$

\begin{tabular}{lrrrr}
\hline \hline Subject & Frequency $^{b}$ & Intensity $^{c}$ & SOA $^{d}$ & Location $^{e}$ \\
\hline 1 & 14 & 4 & 70 & 15 \\
2 & 40 & 12 & 180 & 15 \\
3 & 50 & 10 & 150 & 30 \\
4 & 26 & 3 & 100 & 15 \\
5 & 60 & 5 & 170 & 15 \\
6 & 6 & 3 & 90 & 15 \\
7 & 14 & 5 & 120 & 15 \\
8 & 26 & 5 & 130 & 15 \\
& & & & \\
\hline
\end{tabular}

Note: $\mathrm{SOA}=$ stimulus-onset asynchrony.

${ }^{a}$ The lack of variability in the location dimension is probably due to a limitation of our loudspeaker setup, which did not allow us to get the standard location loudspeaker and the deviant location loudspeakers closer together than $15^{\circ}$. This amount of deviance was detectable above the criteria by all but one subject. ${ }^{b}$ Decrement from $932 \mathrm{~Hz} .{ }^{c}$ Decrement from $70 \mathrm{~dB}$. ${ }^{d}$ Decrement from $500 \mathrm{~ms} .{ }^{e}$ Degrees toward the middle.

and on their right side in the second session. The order was reversed for the other four subjects.

The subjects were seated in a reclining chair in a soundattenuated and electrically shielded chamber with a reverberation time of less than $0.1 \mathrm{~s}$ at $1000 \mathrm{~Hz}$ (IAC, Model 403-A). They were instructed to watch a nature movie presented silently on a computer monitor, and ignore the sounds. The part of the screen where the movie was presented extended a visual angle of $5.7^{\circ}$, at a distance of $100 \mathrm{~cm}$ from the subject's eyes. Ten blocks of 500 stimuli were presented, with a few minutes break between blocks. In each block, 400 tones ( $80 \%$ ) were standard whereas the remaining 100 stimuli were deviants, equally divided between the four types of deviance. Thus, the probability of each deviant type was .05. Standard and deviant tones were presented in a pseudorandom order (different for each subject and block) with the constraint that at least three consecutive standard tones preceded each deviant tone.

\section{EEG Recording and Averaging}

The EEG was recorded from 32 tin electrodes referenced to the tip of the nose. The recording sites were based on the 10-20 system with 12 additions (FT7, FC5, FC3, TP7, CP5, Left mastoid, FT8, FC6, FC4, TP8, CP6, Right mastoid). All electrodes were mounted on a custom-made cap (ECI), except for the mastoid electrodes. The electrooculogram (EOG) was recorded with two electrodes, one located at the outer cantus of the right eye and the other at the infraorbital region of the same eye.

The EEG was continuously sampled at $250 \mathrm{~Hz}$, amplified $\times 20,000$ with an analog band-pass filter of $0.1 \mathrm{~Hz}$ to $100 \mathrm{~Hz}$, and stored for off-line analysis. For ERP averaging, the EEG was divided into 550-ms epochs starting $80 \mathrm{~ms}$ before the stimulus. Epochs with EEG or EOG exceeding $\pm 100 \mu \mathrm{V}$ were excluded from the averaging. The epochs were averaged separately for each stimulus type. The baseline was adjusted by subtracting the mean amplitude of the prestimulus period of each ERP from all the data points in the epoch. Frequencies lower than $1 \mathrm{~Hz}$ or higher than 30 $\mathrm{Hz}$ ( $-3 \mathrm{~dB}$ points) were digitally filtered out from the ERPs after averaging.

\section{Data Analysis}

MMN properties were measured on difference waveforms calculated by subtracting the ERPs elicited by standard trials from those 
elicited by deviant trials in the same block. The amplitude of the MMN was assessed in two ways: (a) by measuring the maximum negativity at Fz between 100-250 ms, in each subject, and (b) by averaging the amplitude within an epoch of $12 \mathrm{~ms}$ before and 12 ms after the peak latency of the MMN in the grand-averaged difference waveforms, across a predesignated cluster of electrodes (Fz, F3, F4, FC3, FC4, FC5, FC6, F7, F8, FT7, FT8). Hence, whereas the first measure was evidence of the most negative peak in each subject, the second disregarded individual variation of peak latency and summated across a larger scalp distribution. In addition, topographical analyses were done testing the interaction across all 32 electrodes and the different conditions. All statistical analyses were done within subjects. The significance of the difference between the response to the standard and to the deviant was as- sessed for each dimension and side using one-tailed paired $t$ tests. Differences among conditions were validated by analysis of variance (ANOVA).

\section{Results}

Visual inspection of the ERPs elicited by the standard tones and the four types of deviant stimuli revealed a negative shift of the deviants compared with the standards between 100 and $250 \mathrm{~ms}$ (Figure 1). This MMN is clearly seen in the difference waveforms, computed by subtracting the waveform elicited by the standard event from that elicited by each type of deviance (Figure 2).

Regardless of dimension or side of stimulation, the MMN was most prominent at the frontal electrodes and less so centrally. The

\section{Grand Average Waveforms Stimuli on Left Stimuli on Right}
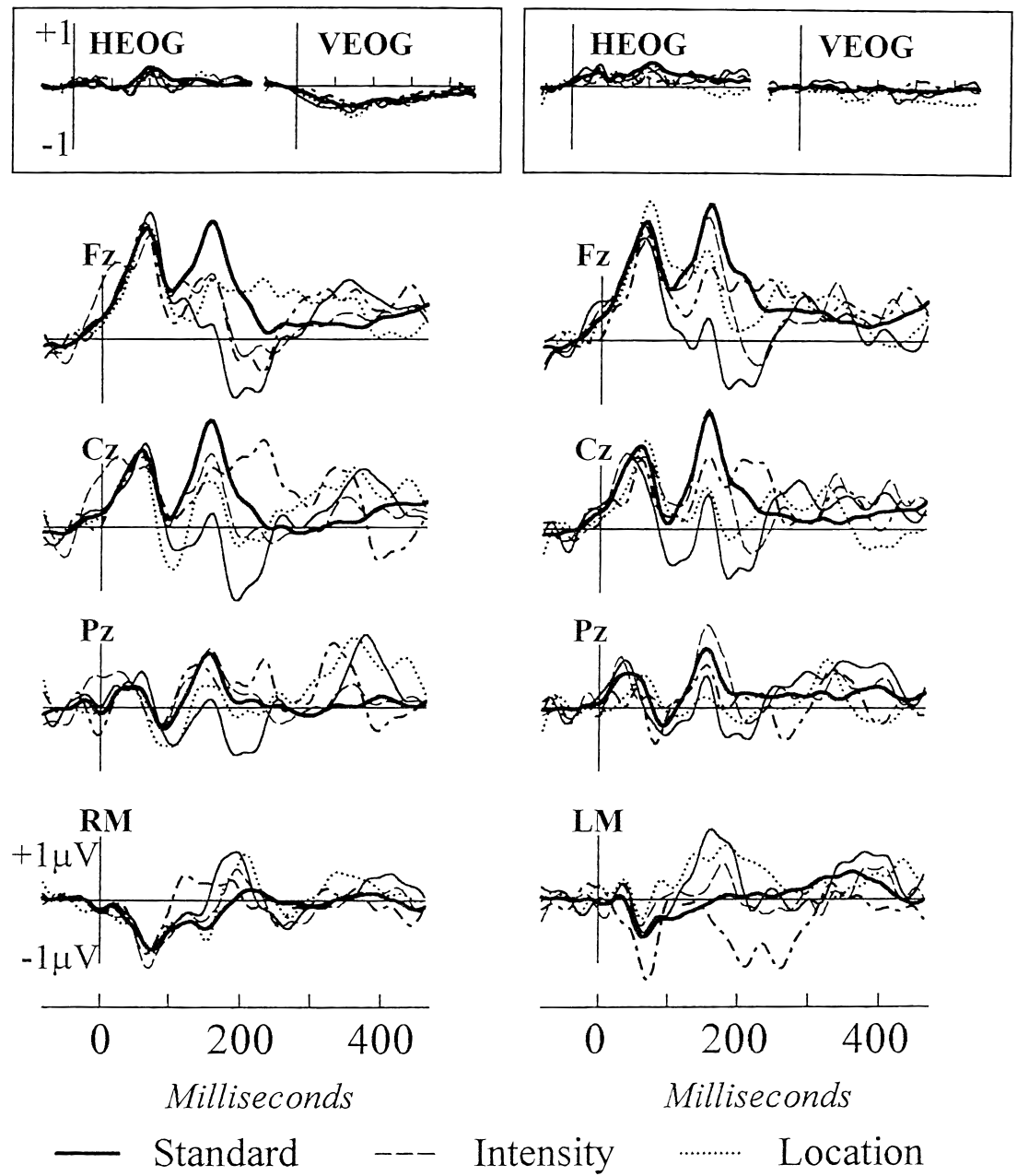

Figure 1. Grand average event-related potentials (ERPs) elicited by the standard tones and each of the four types of deviant tones. The left mastoid (LM) is shown for right side presentation, and the right mastoid (RM) is shown for left side presentation. The horizontal bar represents an epoch from $80 \mathrm{~ms}$ before to $470 \mathrm{~ms}$ after the stimulus, and the vertical bar $\pm 1 \mu \mathrm{V}$ for the scalp as well as the electrooculogram (EOG) waveforms. The apparent shift of $\mathrm{N} 1$ toward positive absolute values is probably a consequence of a slow $(1-1.5 \mathrm{~Hz})$ wave overriding the data. This shift can be corrected by high-pass filtering above $1.5 \mathrm{~Hz}$. However, to avoid excessive manipulation of the data, the analysis and plots were performed using the more commonly used high-pass filter at $1.0 \mathrm{~Hz}$. 


\section{Difference Waves: Deviant -Standard Stimuli on Left Stimuli on Right}
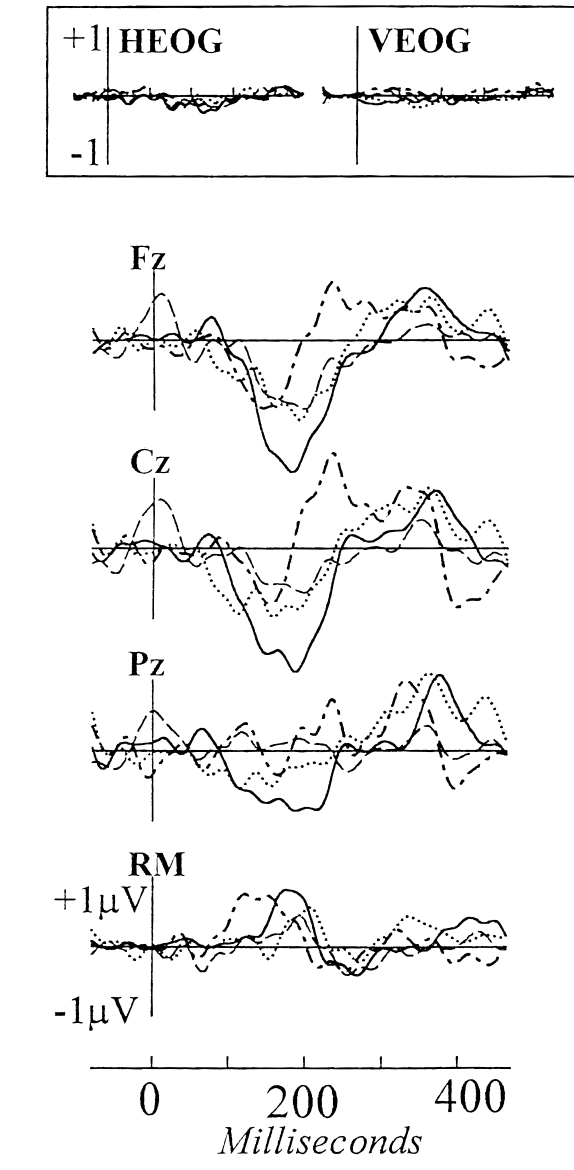

- Frequency ---Intensity
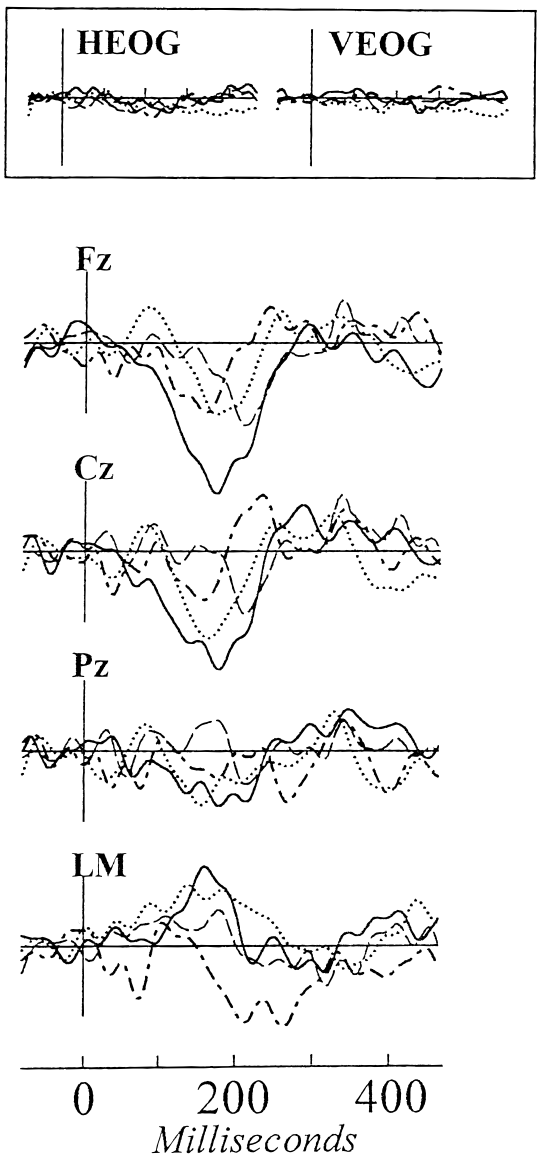

-................SOA

Figure 2. Grand average difference waves computed by subtracting the response to the standard stimuli from the response to each type of deviant. See Figure 1 for details.

MMN was accompanied by a positivity (polarity reversal) at the mastoid sites (Figures 2 and 3). Following the terminology used by Schröger (1995), the MMN elicited by frequency, intensity, SOA, and location shifts will be referred to hereinafter as MMN-F, MMN-I, MMN-S, and MMN-L, respectively. The effect of the "side of presentation" and "dimension of deviance" on MMN was analyzed using amplitude, peak latency, and spatial distribution as the dependent variables in separate analyses.

\section{Amplitude}

Effects of dimension of deviance and side of stimulation. The amplitude of the MMN, averaged over the cluster of frontal electrodes, was significantly different from zero for all the dimensions, whether the stimuli came from the right or the left side of the subject (Table 2).

For individually measured peak amplitudes (at Fz), the effect of dimension-of-deviance was significant, $F(3,21)=4.23, p<.02$, G-G $\epsilon=0.70$, whereas the effect of side-of-deviance was not, $F(1,7)<1.0$. The interaction between the two effects was also not significant, $F(3,21)<1.00$. Post hoc univariate comparisons between dimensions (collapsed over right and left side stimulation) revealed that MMN-F was larger than all the other types of MMN, $F(1,7)=8.09, p<.025$, which did not differ among themselves. When the same type of analysis was done using average amplitude at the frontal cluster of electrodes around the grand-average peak latency (rather than the individual most negative peak), no significant main effects or interaction were found. Therefore the numerical results of the statistical analyses reported below refer only to the individual maximum negativity at Fz. The same analyses were run, however, using the average amplitude measure as the dependent variable, and whenever qualitative differences were found between the two types of analysis, both are reported.

Correlation between the MMN amplitude and the objective size of deviance. Correlation analyses were conducted to test the relations between the individual magnitudes of objective deviance and the MMN separately for frequency, intensity, and SOA. Correlations were not computed for location deviance due to the lack of 


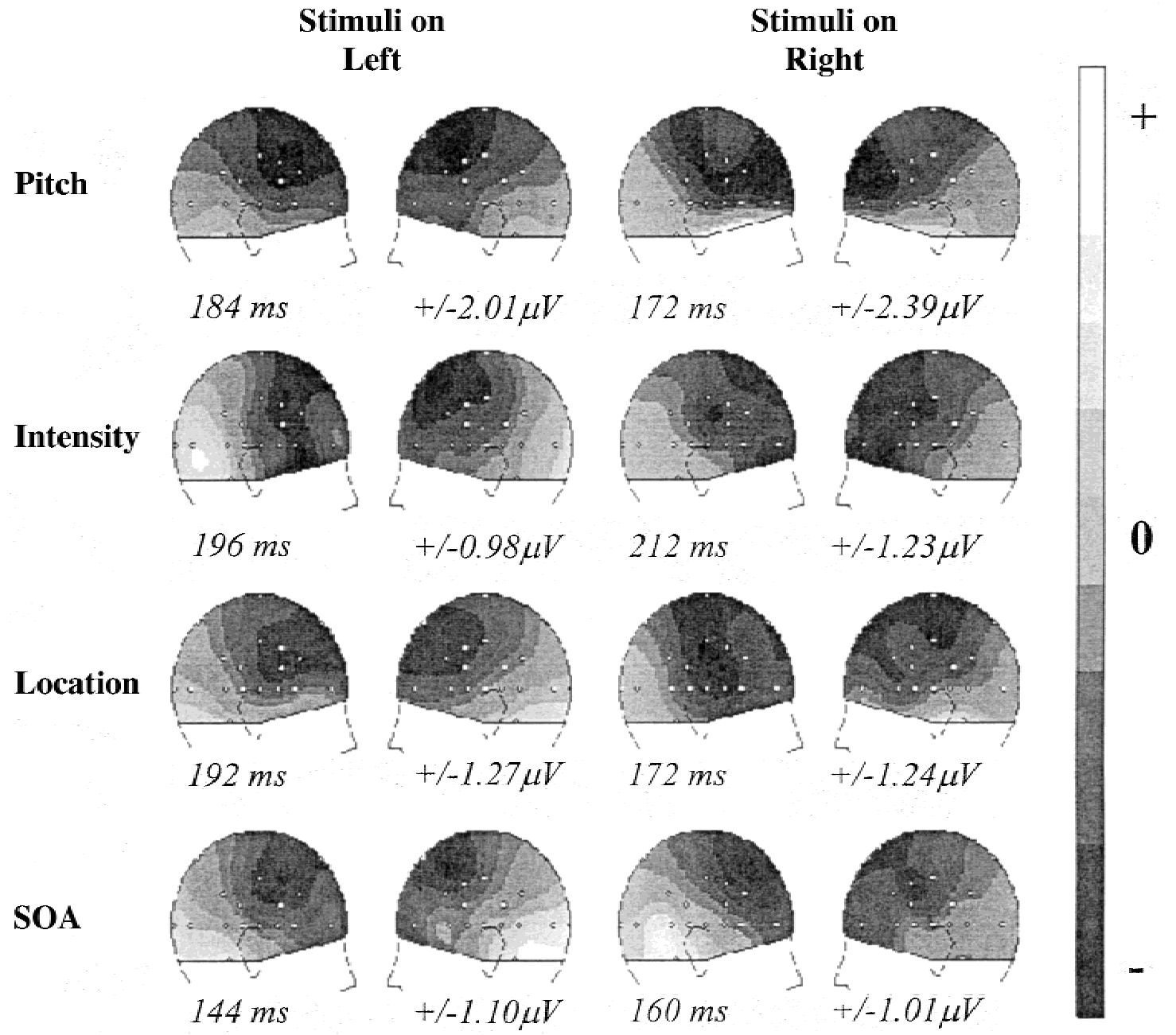

Figure 3. Lateral views of scalp voltage distribution (spline interpolation) at the peak amplitude of the mismatch negativity (MMN) elicited by the four types of deviance. Darker regions represent more negative potentials. White dots represent electrode locations seen from these lateral viewpoints.

variability in the magnitude of deviance in this dimension. The correlation between the magnitude of frequency deviance and the amplitude of MMN-F was marginally significant (Spearman's $r=$ $.65, p<.05)$. Although the magnitudes of SOA and intensity deviance were also positively correlated with the amplitude of MMN, these correlations were not statistically significant (Spearman's $r$ for MMN-I: .53, MMN-S: .62; critical value for .05 probability of error: .64).

Table 2. Grand Average Peak Mismatch Negativity Latencies, Amplitudes, Standard Errors, $t$ Value, and Probability of Type I Error (One-Tailed) ${ }^{a}$

\begin{tabular}{|c|c|c|c|c|c|c|c|c|}
\hline & \multicolumn{2}{|c|}{ Frequency } & \multicolumn{2}{|c|}{ Intensity } & \multicolumn{2}{|c|}{ SOA } & \multicolumn{2}{|c|}{ Location } \\
\hline & Right & Left & Right & Left & Right & Left & Right & Left \\
\hline Latency (ms) & 183 & 178 & 211 & 179 & 141 & 146 & 177 & 185 \\
\hline Mean amplitude (SE) & $\begin{array}{c}-1.61 \\
(0.47)\end{array}$ & $\begin{array}{c}-1.54 \\
(0.38)\end{array}$ & $\begin{array}{c}-0.88 \\
(0.25)\end{array}$ & $\begin{array}{c}-0.78 \\
(0.14)\end{array}$ & $\begin{array}{c}-0.57 \\
(0.24)\end{array}$ & $\begin{array}{c}-0.69 \\
(0.26)\end{array}$ & $\begin{array}{c}-0.95 \\
(0.19)\end{array}$ & $\begin{array}{c}-0.99 \\
(0.25)\end{array}$ \\
\hline $\mathrm{t}(7)$ & $\begin{array}{c}-3.44 \\
p<.006\end{array}$ & $\begin{array}{c}-4.00 \\
p<.003\end{array}$ & $\begin{array}{c}-3.52 \\
p=.005\end{array}$ & $\begin{array}{c}-5.76 \\
p<.001\end{array}$ & $\begin{array}{c}-2.41 \\
p<.025\end{array}$ & $\begin{array}{c}-2.65 \\
p<.02\end{array}$ & $\begin{array}{c}-5.03 \\
p=.001\end{array}$ & $\begin{array}{c}-3.94 \\
p=.003\end{array}$ \\
\hline
\end{tabular}

Note: $\mathrm{SOA}=$ stimulus-onset asynchrony.

${ }^{a}$ Amplitudes are the average amplitudes $\pm 12 \mathrm{~ms}$ around the grand average peak MMN latency at Fz. 
Correlation between right and left side of stimulation. Because the responses to stimuli presented on the right or on the left were recorded on separate days and no general effects of side were found in any dimension, we considered the two recordings a kind of test-retest reliability measure and analyzed it by calculating the correlation between the two sets of data. The amplitudes of MMN-F elicited by left and right side tones were significantly correlated (Spearman's $r=.90, p<.01$ ). In contrast, the same type of correlation was not significant for the other types of MMN (Spearman's $\mathrm{r}$ for MMN-I: -.11, MMN-S: .51, MMN-L: .43; critical value for .05 probability of error: .64)

Correlation between dimensions. An additional analysis tested the correlation between the amplitudes of MMN elicited by the four investigated dimensions. When the dependent variable was the MMN at $\mathrm{Fz}$ measured at the individual peak negativity, only the correlation between MMN-F and MMN-I was significant (Spearman's $r=.67, p<.05)$. No significant correlation among the MMN types was found when the dependent variable was the averaged frontal negativity around the grand average peak negativity.

\section{Latency}

Effects of dimension of deviance and side of stimulation. The peak latency of the MMN elicited by MMN-S was the shortest, followed by MMN-L and MMN-F, and finally by MMN-I, which elicited the latest MMN (Table 2). The ANOVA of the latency of the most negative peak between 100-250 ms, with side of stimulation and dimension of deviance as factors, revealed a significant main effect of dimension, $F(3,21)=8.05, p<.001$, G-G $\epsilon=$ .81 , and a significant interaction between side and dimension, $F(3,21)=4.4, p<.015, \mathrm{G}-\mathrm{G} \epsilon=.62$. A post hoc univariate analysis of the effect of dimension revealed that the peak latencies of MMN-F, MMN-I, and MMN-L were not significantly different, whereas the latency of MMN-S was significantly shorter than that of the three other types of MMN, $F(1,7)=17.5, p<.004$. A post hoc analysis of the interaction effect revealed that the interaction resulted from a significantly shorter latency of MMN-I when stimuli were presented on the left rather than on the right side, $F(1,7)=7.34, p<.05$, whereas the other types of MMN were not affected by the side of stimulation (Figure 4).

\section{MMN peak latency at $\mathrm{Fz}$ (in milliseconds)}

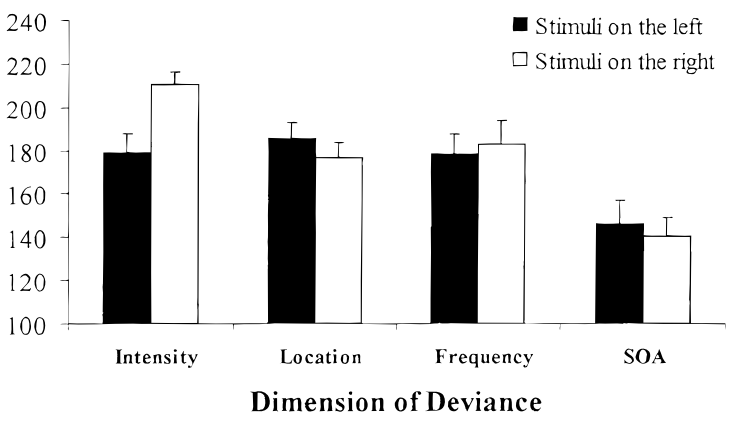

Figure 4. Average of individual subjects' peak latencies of mismatch negativity (MMN) elicited by the four types of deviance used. Tones were presented from loudspeakers on the left of the subject (dark bars) or on the right of the subject (light bars). The error bars represent the standard error of the mean.
Correlation between the MMN latency and the objective size of deviance. A significant negative correlation was found between the magnitude of frequency deviance and the latency of MMN-F (Spearman's $r=-.86, p<.01$ ). Because the latency of MMN-I depended on the side from which the tones were presented (see the previous paragraph), we repeated the analysis separately for the MMNs elicited by tones presented on the left and on the right side of the subject. This analysis revealed a significant correlation between the objective magnitude of intensity deviance and MMN-I only when the tones were presented to the left of the subject (Spearman's $r=-.75, p<.05$ ). MMN-F was correlated with the degree of frequency deviance whether the tones were presented to the left or to the right of the subject $(r=-.89, p<.01$ and $r=-.77, p<.05$, respectively) .

Correlation between right- and left-side stimulation. There was a significant positive correlation between the latencies of MMN-F elicited by tones presented to the right and the left of the subject (Spearman's $r=.95, p<.01$ ). However, the same correlations for MMN-I $(r=-.11)$, MMN-S $(r=.49)$, and MMN-L (0.53) were not statistically significant.

Correlation between dimensions. No significant correlation was observed between the latencies of MMN elicited by any two dimensions of deviance.

\section{Spatial Distribution of Scalp Potentials}

Figure 3 displays spline interpolation of the potentials in the different conditions, at each respective peak MMN latency. It is evident that in all conditions, and over both hemispheres, the MMN is charac-terized by a frontal negativity, accompanied by positivity at the mastoid and posterior temporal sites. The frontal negativity tended to be stronger over the right hemisphere, regardless of side of stimulation, when the deviance was in the location dimension (MMN-L). With the other types of MMN, however, no clear asymmetry was observed. The maps also show that the orientation of the "zero line," which demarcates the region of negativity from the region of positivity, varied with the type of MMN. It was the most horizontal for MMN-F (roughly extending from the inion to the auricular trigon), the most vertical for MMN-I (roughly between Fz and the trigon), and midway for MMN-S and MMN-L. These differences in spatial distribution were not significant, however, in ANOVA with all electrodes and dimensions of deviance as factors, following normalization using McCarthy and Wood's (1985) method, $F(93,651)<1$ for the interaction between dimension and electrodes. The voltage distribution and the orientation of the zero line were symmetrical across hemispheres and similar within dimensions, whether the MMN was elicited by presenting the tones to the subject's right or left sides.

\section{Discussion}

The purpose of the present study was to compare the MMN elicited by deviance in different dimensions, while holding extraneous variables as constant as possible. Previous studies compared MMN elicited by manipulating different auditory dimensions. In most of these publications, however, differences in individual performance among dimensions have not been reported and, when reported (e.g., Schröger, 1995), they were large. Therefore, whereas these studies suggested that mismatch in different physical dimensions of the auditory stimulus may elicit distinct neuronal activities (e.g., Deacon et al., 1998; Giard et al., 1995; Levänen, Ahonen, Hari, McEvoy, \& Sams, 1996; Levänen, Hari, McEvoy, \& Sams, 1993; 
Schröger, 1995), they did not allow a simple comparison of the MMN patterns per se across deviant types. In the present study we used individually adjusted parameters to determine the size of deviance, and mixed all the deviant stimuli along four dimensions in the same block. The results revealed that, despite the comparable psychophysical performance level, there were significant differences in the MMN amplitude, latency, and variability across the four dimensions. These differences, however, were not constant across subjects, suggesting heterogeneity in the manner of processing different auditory dimensions among subjects. The side at which the stimuli were delivered did not have a significant main effect.

The average amplitude of MMN elicited by deviance in frequency was higher than the amplitudes of the MMNs elicited by deviance in intensity, location, and SOA, which were remarkably close to one another. This pattern suggests that despite the comparable subjective distinctiveness of deviance across all four dimensions, the frequency deviance may have been more obtrusive to the system than the other types of deviance. In this respect it is interesting to note that the difference between MMN-F and the other types of MMN was evident at $\mathrm{Fz}$ but not at the mastoid. Thus, it is possible that the difference between frequency and other dimensions is related to the putatively attention-related frontal generator of MMN (cf., Deouell, Bentin, \& Giard, 1998; Giard, Perrin, Pernier, \& Bouchet, 1990), rather than to the auditory cortex generators in the supratemporal plane that are probably better reflected by the mastoid positivity. On the other hand, whereas this frontal component of MMN has been suggested to be related to involuntary attention switch, the stronger MMN at Fz was not associated with a more prominent $\mathrm{P} 3 \mathrm{a}$, which is assumed to be a sign of such a switch (Näätänen, 1992).

The fact that the MMN-F amplitude was greater than the amplitude of the other MMN types may also result from the different orientation of the current dipoles underlying the different types of MMN. For example, in a recent study, the equivalent current dipole (ECD) that best fitted the potential distribution of MMN-I was oriented along a lower-postero-medial to upper-antero-lateral plane, whereas the ECD associated with MMN-F was oriented along a lower-postero-lateral to higher-antero-medial (Giard et al., 1995). Therefore, it may be expected that the MMN-F dipole, pointing toward the anterior midline, would be better detected at Fz than the MMN-I dipole which points away from the midline. Although the difference in spatial distribution of the four types of MMN was not statistically significant in our study, the different orientations of the isopotential zero-lines resembled that observed in Giard et al.'s study. The absence of statistical significance in the current study may have been caused by the considerably fewer trials used in our study compared with the study of Giard et al.

Like the MMN's amplitude, the latency of the MMN varied across dimensions. However, whereas the MMN-F was outstanding for amplitude, for latency the MMN-S differed significantly from all other types of MMN by having a shorter latency. Because in our design the magnitude of deviance in SOA was equivalent to the other types of deviance, the shorter latency elicited cannot be explained by the reported linear relationship between the magnitude of deviance and the latency of MMN (Tiitinen et al., 1994). The present results suggest, therefore, that detection of an earlierthan-usual stimulus may be faster than the detection of the other kinds of deviation tested.

The MMN-L latency in our study was apparently longer than that reported in previous studies (e.g., Kujala, Alho, Paavilainen, Summala, \& Näätänen, 1992; Paavilainen et al., 1989). This dif- ference might have had one of two causes: One cause might be that the location shift was smaller $\left(15^{\circ}\right)$ in the present study than in those previous studies. The other cause might be that we used a free-field setup, whereas the shorter MMN-L latencies were recorded with apparent shifts introduced by interaural time or intensity differences. Supporting our explanation, when a free-field setup was used, and the shift in location was comparable to ours $\left(10^{\circ}\right)$, the latency of MMN-L was even slightly longer than ours (Paavilainen et al., 1989).

The similar latency of the MMN-L and MMN-F in the present study seems to be at odds with results published by Schröger and his associates in a series of studies. For example, using frequency change from $600 \mathrm{~Hz}$ to $625 \mathrm{~Hz}$, and location shift created by interaural time delay through earphones, Schröger (1995) found an earlier MMN-L than MMN-F (but did not find any amplitude differences). However, the comparison between the two types of MMN in that study may not have been valid because the hit rates for the two types of deviants were not only significantly different, but also varied widely across subjects (27-96\% for frequency deviants and $1-93 \%$ for location deviants). In a more recent study, Schröger and Wolff (1997) replicated the latency difference between the MMN-L and MMN-F and reported that the hit rate and reaction time for the discrimination of the deviants and standards was similar for the two dimensions. This result, however, was obtained in a control group, different from the group tested in the MMN study. Moreover, the objective difference between the standards and the deviants in Schröger and Wolff's study was large (from 600 to $660 \mathrm{~Hz}$ and from $45^{\circ}$ to the right, to $45^{\circ}$ to the left). Notably, although in the present study the deviance in location was much smaller $\left(15^{\circ}\right)$, the subjects' performance was almost perfect. Therefore, it is possible that the discrimination between standards and deviants in the Schröger and Wolff study (especially for the deviance in location) was too easy to reveal differences between the subjective discrimination for frequency and location. ${ }^{2}$ Nonetheless, their results led Schröger and Wolff to suggest that preattentive information about stimulus location is stored early, and forms a substrate for later selective spatial attention mechanisms. The comparable latency of MMN-L and MMN-F in the present study casts doubt on this interpretation, suggesting that when discrimination performance levels are equated individually across dimensions, the difference in latency does not hold, at least in a (more natural) free-field setting. At the very least, this discrepancy highlights the difficulty of drawing conclusions from comparisons between dimensions and the relevance of individual performance data when such comparisons are the goal.

The amplitude of the MMN-F and its latency were distinct from those elicited by other dimensions also by significantly correlating with the objective physical difference between the standards and the deviants. Similar correlations were reported in previous studies (e.g., Tiitinen et al., 1994). In those studies, however, the magnitude of deviance was manipulated within the subject. Consequently, the increase of the actual magnitude of deviance in those studies enhanced the subjective salience of the deviant stimuli. In contrast, the magnitude of deviance in our study was varied between subjects, while keeping the subjective salience level presumably equal. The possibility that this effect was due to an overlap

\footnotetext{
${ }^{2}$ Indeed, in Schröger and Wolff (1997), the hit rate was not at ceiling. However, in that study the time during which subjects' responses were accepted was very limited and many correct responses could conceivably have been rejected on the basis of being too late (Schröger, personal communication, 1998).
} 
of an enhanced $\mathrm{N} 1$ in response to the different frequency of the deviant is unlikely, considering both the minute changes in frequency between the standard and deviant stimuli (from 0.6 to $6 \%$ ), and the distance of the MMN latency $(180 \mathrm{~ms})$ from the latency of N1 (100 ms). Hence, unlike previous studies that emphasized the effect of subjective discriminability on the MMN amplitude (e.g., Kraus et al., 1993; Lang et al., 1990; Näätänen, Schröger, et al., 1993, Sams et al., 1985), inasmuch as the present attempt to equate the salience level was successful, and keeping in mind the small sample tested, our study suggests that the absolute frequency difference between standard and deviant stimuli plays a role in determining the amplitude of the MMN.

Although there was no overall trend toward side-of-stimulation effects on the amplitude of MMN, the only condition in which MMNs elicited by right and left side stimulation were significantly correlated was the frequency deviance. This finding was true for the latency and amplitude variables. As stimulation on the left side and the right side were conducted in separate sessions several days apart, the lack of correlation should probably be taken as a poor test-retest reliability at the individual level of all the types of MMN that we tested, with the exception of MMN-F. Both Escera and Grau (1996), who tested MMN-F replicability between two sessions held $2 \mathrm{hr}$ apart, and Pekkonen et al. (1995), who tested replicability over 1 month, reported good replicability at the group level, but not at the individual level. These authors suggested that the poorer replicability at the individual level is a consequence of using an insufficient number of deviant tones (resulting in a low signal-to-noise ratio in the average). In our study, there were more deviants (250 of each kind) than in either the Escera and Grau (1996) or the Pekkonen et al. (1995) study (160 and 150, respectively). Therefore, this factor may explain the improved individuallevel test-retest replicability obtained for MMN-F here. However, there are two additional factors that may also have enhanced the reliability of the MMN-F's amplitude and latency in our study.
These factors were the low probability of deviants (.05 for each type of deviance) and the fact that we assured that the present magnitude of deviance was within the subjects' discrimination capacity. Nonetheless, even under these more optimal conditions, the individual test-retest reliability of MMN-L, MMN-I, and MMN-S was not sufficiently augmented. If indeed the problem is extrinsic (i.e., resulting from a low signal-to-noise ratio) rather than intrinsic (i.e., resulting from a low reliability of the processes underlying $\mathrm{MMN}$ ), then more experimentation will probably reveal the conditions for its resolution.

\section{Summary and Conclusions}

The purpose of this study was to compare the MMN elicited by deviance along the dimensions of frequency, intensity, SOA, and location. The MMN elicited by these types of deviance varied significantly in amplitude, latency, and reliability, despite the fact that discrimination between the standards and the deviants was comparably easy for the different types of deviance. Specifically, the amplitude of MMN-F was larger than all other types of MMN, whereas the latency of MMN-S was shorter than the latency of any other MMN type. Conversely, and in contrast to previous reports, there was no difference in latency between MMN-L and MMN-F under the current conditions, emphasizing the importance of individual discrimination measurements when comparisons between dimensions are sought. Moreover, when the individual's discrimination level was controlled for, the within-subject test-retest reliability for MMN-F was better than previously reported. On the other hand, whereas the reliability at the group level was good for all dimensions, the within-subject reliability in dimensions other than frequency was still low. The relatively large intersubject variability across dimensions, and the weak test-retest reliability in some dimensions require the use of utmost caution when employing the MMN in single case studies.

\section{REFERENCES}

Aaltonen, O., Niemi, P., Nyrke, T., \& Tuhkanen, M. (1987). Event related brain potentials and the perception of a phonetic continuum. Biological Psychology, 24, 197-207.

Böttcher-Gandor, C., \& Ullsperger, P. (1992). Mismatch negativity in eventrelated potentials as a function of varying inter-stimulus intervals. Psychophysiology, 29, 546-550.

Deacon, D., Nousak, J., Pilotti, M., Ritter, W., \& Yang, C. M. (1998). Automatic change detection: Does the auditory system use representations of individual stimulus features or gestalts? Psychophysiology, 35, 413-419.

Deouell, L. Y., Bentin, S., \& Giard, M. H. (1998). Mismatch negativity in dichotic listening: Evidence for interhemispheric differences and multiple generators. Psychophysiology, 35, 355-365.

Escerea, C., \& Grau, C. (1996). Short-term replicability of the mismatch negativity. Electroencephalography and Clinical Neurophysiology, 100, $549-554$.

Giard, M. H., Lavikainen, J., Reinikainen, K., Perrin, F., Bertrand, O., Pernier, J., \& Näätänen, R. (1995). Separate representation of stimulus frequency, intensity, and duration in auditory sensory memory: An event related potential and dipole-model analysis. Journal of Cognitive Neuroscience, 7, 133-143.

Giard, M. H., Perrin, F., Pernier, J., \& Bouchet, P. (1990). Brain generators implicated in the processing of auditory stimulus deviance: A topographic event related potential study. Psychophysiology, 27, 627-640.

Kane, N. M., Curry, H., Buttler, S. R., \& Cummins, B. H. (1993). Electrophysiological indicators of awakening from coma. Lancet, 341, 688.

Korpilahti, P., \& Lang, H. (1994). Auditory ERP components and mismatch negativity in dysphasic children. Electroencephalography and Clinical Neurophysiology, 91, 256-264.
Kraus N., \& McGee, T. (1994). Mismatch negativity in the assessment of central auditory function. American Journal of Audiology, 3, 139-151.

Kraus, N., McGee, T., Micco, A., Carrell, T., Sharma, A., \& Nicol, T. (1993). Mismatch negativity in school-age children to speech stimuli that are just perceptibly different. Electroencephalography and Clinical Neurophysiology, 88, 123-130.

Kujala, T., Alho, K., Paavilainen, P., Summala, H., \& Näätänen, R. (1992). Neural plasticity in processing of sound location by the early blind: An event-related potential study. Electroencephalography and Clinical Neurophysiology, 84, 469-472.

Lang, A. H., Eeorola, O., Korpilahti, P., Holopainen, I., Salo, S., \& Aaltonen, O. (1995). Practical issues in the clinical application of mismatch negativity. Ear and Hearing, 16, 118-130.

Lang, A. H., Nyrke, T., Ek, M., Aaltonen, O., Raimo, I., \& Näätänen, R. (1990). Pitch discrimination performance and auditive event-relatedpotentials. In C. H. M. Brunia, A. W. K. Gaillard \& A. Kok (Eds.), Psychophysiological brain research (Vol. 1, pp. 294-298). Tilburg: Tilburg University Press.

Levänen, S., Ahonen, A., Hari, R., McEvoy L., \& Sams, M. (1996). Deviant auditory stimuli activate human left and right auditory cortex differently. Cerebral Cortex, 6, 288-296.

Levänen, S., Hari, R., McEvoy, L., \& Sams, M. (1993). Responses of the human auditory cortex to changes in one versus two stimulus features. Experimental Brain Research, 97, 177-183.

McCarthy, G., \& Wood, C. C. (1985). Scalp distribution of event-related potentials: An ambiguity associated with analysis of variance models. Electroencephalography and Clinical Neurophysiology, 62, 203-208.

Näätänen, R. (1990). The role of attention in auditory information processing as revealed by event-related potentials and other brain measures of cognitive function. Behavioral and Brain Sciences, 13, 201-288. 
Näätänen, R. (1992). Attention and brain function. Hillsdale, NJ: Erlbaum.

Näätänen, R., Jiang, D., Lavikainen, J., Reinikainen, K., \& Paavilainen, P. (1993). Event-related potentials reveal a memory trace for temporal features. NeuroReport, 5, 310-312.

Näätänen, R., Paavilainen, P., Alho, K., Reinikainen, K., \& Sams, M. (1987). The mismatch negativity to intensity changes in an auditory stimulus sequence. In R. Johnson, Jr., J. W. Rohrbaugh, \& R. Parasuraman (Eds.), Current trends in event related potential research (EEG suppl. 40, pp. 125-131). Amsterdam: Elsevier.

Näätänen, R., Paavilainen, P., Alho, K., Reinikainen, K., \& Sams, M. (1989). Do event-related potentials reveal a mechanism of the auditory sensory memory in the human brain? Neuroscience Letters, 98, 217 221.

Näätänen, R., Schröger, E., Tervaniemi, M., Karakas, S., \& Paavilainen, P. (1993). Development of memory trace for complex sound patterns in the human brain. NeuroReport, 4, 503-506.

Paavilainen, P., Alho, K., Reinikainen, K., Sams, M., \& Näätänen, R. (1991). Right hemisphere dominance of different mismatch negativities. Electroencephalography and Clinical Neurophysiology, 78, 466479.

Paavilainen, P., Jiang, D., Lavikainen, J., \& Näätänen, R. (1993). Stimulus duration and the sensory memory trace: An event-related potential study. Biological Psychology, 35, 139-152.

Paavilainen, P., Karlsson, M. L., Reinikainen, K., \& Näätänen, R. (1989). Mismatch negativity to change in spatial location of an auditory stimulus. Electroencephalography and Clinical Neurophysiology, 73, 129141.

Pekkonen, E., Rinne, T., \& Näätänen, R. (1995). Variability and replicability of mismatch negativity. Electroencephalography and Clinical Neurophysiology, 96, 546-554.
Ritter, W., Deacon, D., Gomes, H., Javitt, D. C., \& Vaughan, H. G. (1995). The mismatch negativity of event-related potentials as a probe of transient auditory memory: A review. Ear and Hearing, 16, 52-57.

Sams, M., Paavilainen, P., Alho, K., \& Näätänen, R. (1985). Auditory frequency discrimination and event-related potentials. Electroencephalography and Clinical Neurophysiology, 62, 437-448.

Schröger, E. (1994). Automatic detection of frequency change is invariant over a large intensity range. NeuroReport, 5, 825-828.

Schröger, E. (1995). Processing of auditory deviants with changes in one versus two stimulus dimensions. Psychophysiology, 32, 55-65.

Schröger, E. (1996). The influence of stimulus intensity and inter-stimulus interval on the detection of pitch and loudness changes. Electroencephalography and Clinical Neurophysiology, 100, 517-526.

Schröger, E., Tervaniemi, M., \& Näätänen, R. (1995). Time course of loudness in tone patterns is automatically represented by the human brain. Neuroscience Letters, 202, 117-120.

Schröger, E., \& Wolff, C. (1997). Fast preattentive processing of location: A functional basis for selective listening in humans. Neuroscience Letters, 232, 5-8.

Tiitinen, H., May, P., Reinikainen, K., \& Näätänen R. (1994). Attentive novelty detection in humans is governed by pre-attentive sensory memory. Nature, 372, 90-92.

(Received January 7, 1998; ACCEPted May 27, 1998) 